\title{
Effects of Methylmethionine Sulfonium Chloride on Activity and Tight Junction Protein Expression of Intestinal Porcine Jejunum Epithelial Cells (IPEC-J2)
}

\author{
Jianping Yang ${ }^{1,2 \#}$, Xinfeng $\mathrm{Li}^{2,3 \#}$, Xinlei Wang ${ }^{1}$, Xin Wen ${ }^{1}$, Tongtong Zhang ${ }^{3}$, Wenjie Xu ${ }^{3}$
}

10.18805/IJAR.B-1384

\begin{abstract}
Background: The intestinal mucosal epithelium acts as a physical and biochemical barrier and plays an important role in regulating of barrier function and immune homeostasis. Methylmethionine sulfonium chloride (MMSC) is a multifaceted amino acid that is critical to the normal physiology of the gastrointestinal tract. The present study investigated the effects of extracellular MMSC on intestinal epithelial cell line (IPEC-J2).

Methods: IPEC-J2 cells were treated with $0.1,0.5$ and $1 \mathrm{mM} \mathrm{MMSC}$, respectively for an additional $24 \mathrm{~h}$. CCK-8 assay was used to evaluate cell proliferation. The cell Annexin V-FITC/PI apoptosis were analyzed by flow cytometry (FCM) method. The mRNA transcript and protein expression levels of tight junction proteins in IPEC-J2 cells were detected by real-time quantitative polymerase chain reaction (RT-qPCR) and western blotting (WB).

Result: The results showed that MMSC could stimulate IPEC-J2 cells proliferation and inhibit cell apoptosis. In addition, the RTqPCR and WB results indicated that $0.5 \mathrm{mM} \mathrm{MMSC}$ significantly increased the mRNA and protein expression of tight junction proteins, including occludin, claudin-1 and zonula occludin-1 (Zo-1). These findings may provide valuable information to investigate further the possible mechanism and function of MMCS on the intestinal barrier function.
\end{abstract}

Key words: Intestinal mucosal epithelium, IPEC-J2, MMSC, Protein expression, Tight junction.

\section{INTRODUCTION}

The intestinal mucosal epithelial barrier serves as the first line of defense against the invasion of pathogenic microorganisms and toxic substances in the intestinal tract (Yu and Li 2014). It is formed by the interaction between the epithelial cells and the tight junction proteins, which play an important role in ion transport, selective absorption of essential nutrients, blockade of harmful substances and so on (Barrett 2008). Recently, it is reported that piglet diarrheal and intestinal inflammation is closed to the intestinal mucosal barrier and the function of tight junctions are important for the maintenance and repair of the intestinal mucosal barrier (Guevarra et al. 2018, Yang et al. 2019).

Tight junctions are intercellular adhesion complexes on epithelial cells, which was composed of integral membrane proteins as well as cytosolic adaptor proteins (Roehlen et al. 2020). Occludin, claudins and zonula occluden (Zo), the main transmembrane component of tight junctions proteins, have been implicated in regulating properties and functions of tight junctions (Suzuki and Hara 2009, Zong et al. 2019). Tight junction proteins were believed to be one of the most important components of the intestinal mucosal barrier and played an important role in health and disease (Farkas et al. 2012, Kojima et al. 2003). So far, intestinal porcine jejunum epithelial cells (IPEC-J2) line isolated from the mid-jejunum of a neonatal piglet is established as an in vitro model for porcine infection studies and nutritional studies (Liu et al. 2010, Zakrzewski et al. 2013).
${ }^{1}$ College of Animal Science and Technology, Henan University of Animal Husbandry and Economy, Zhengzhou, 450046, China.

${ }^{2}$ Henan Key Laboratory of Unconventional Feed Resources Innovative Utilization, Henan University of Animal Husbandry and Economy, Zhengzhou, 450046, China.

${ }^{3}$ College of Veterinary Medicine, Henan University of Animal Husbandry and Economy, Zhengzhou, 450046, China.

\#These authors contributed equally to this work.

Corresponding Author: Xinfeng Li, Henan Key Laboratory of Unconventional Feed Resources Innovative Utilization, Henan University of Animal Husbandry and Economy, Zhengzhou, 450046, China. Email: xinfengli@hnuahe.edu.cn

How to cite this article: Yang, J., Li, X., Wang, X., Wen, X., Zhang, T. and Xu, W. (2021). Effects of Methylmethionine Sulfonium Chloride on Activity and Tight Junction Protein Expression of Intestinal Porcine Jejunum Epithelial Cells (IPEC-J2). Indian Journal of Animal Research. DOI: 10.18805/IJAR.B-1384.

Submitted: 15-05-2021 Accepted: 06-08-2021 Online: 03-09-2021

Methylmethionine sulfonium chloride (MMSC), generally known as vitamin $U$, which is a multifaceted amino acid and used as gastroprotective drug in treating injuries or ulcerations of the digestive tract and skin (Kim et al. 2015, Salim 1992). It is reported that MMSC promoted the mucus barrier of rat gastric mucosa, as it improved the amount and immunoreactivity of surface mucus cell-derived mucin (Ichikawa et al. 2009). More recently, studies showed that 
MMSC pre-treatment in female rats might prevent valproic acid-induced renal damage and lung toxicity (GezginciOktayoglu et al. 2016, Oztay et al. 2020). However, little is known about the effect of the MMSC on the intestinal epithelial cell barrier. Therefore, it would be of interest to examine whether supplementation of MMSC modulates the expression of tight junction proteins.

In the present, we aimed to explore the influence of MMSC on IPEC-J2 cells, including the cell viability, as well as the mRNA and protein expression levels of cell tight junction genes. Results showed MMSC displayed an effective activity in promoting the expressions of the key tight junction proteins. These findings laid a foundation to further study the effects of MMCS on intestinal permeability and barrier function in IPEC-J2 cells.

\section{MATERIALS AND METHODS IPEC-J2 cells culture}

IPEC-J2 cells were cultured and maintained in Dulbecco's modified Eagle's medium/nutrient mixture F-12 (DMEM-F12, Solarbio) supplemented with $10 \%$ foetal bovine serum (FBS, Gibco) and penicillin-streptomycin (100 mg/ml; Invitrogen) at $37^{\circ} \mathrm{C}, 5 \% \mathrm{CO}_{2}$ and $95 \%$ relative humidity. When IPEC-J2 cells reached confluence, $0.25 \%$ trypsin was added for digestion and passage and then cells in the logarithmic growth phase were selected for experiments.

\section{Infection of IPEC-J2 cells with MMSC}

IPEC-J2 cells were cultured onto 96 -well plates at $5 \times 10^{4}$ cells/plate or 6 -well plates at $1 \times 10^{6}$ cells/plate and incubated in complete DMEM-F12 containing 10\% FBS. After $24 \mathrm{~h}$ incubation, culture supernatant was discarded and cells were cultured in DMEM-F12 without FBS containing different concentrations of MMSC for an additional $24 \mathrm{~h}$. Untreated cells were used as a negative control. IPEC-J2 cells in group 1 were infected with $0.1 \mathrm{mM} \mathrm{MMSC}$, whereas group 2 and 3 were infected with $0.5 \mathrm{mM}$ MMSC and $1 \mathrm{mM} \mathrm{MMSC}$, respectively. IPEC-J2 cells in 96-well plates were used to estimate cell proliferation and in 6-well plates were collected for cell apoptosis and protein expression. Each sample was repeated with three times.

\section{Cell proliferation analysis}

CCK-8 assay was used to evaluate cell proliferation. Added $10 \mu \mathrm{L}$ CCK-8 solution (Solarbio) to 96 -well plates and continued to incubate for $4 \mathrm{~h}$. Cell proliferation activity (\%) was calculated as: mean OD450 nm of experimental cells / mean OD450 nm of negative control group cells.

\section{Annexin V-FITC/PI apoptosis assay}

IPEC-J2 cells in 6-well plates were collected, digested into single-cell suspensions with EDTA-free trypsin and then subjected to the Annexin V/PI Apoptosis Detection kit (Invitrogen) for staining according to the manufacturer's instructions. The stained cells were analyzed by flow cytometry (FCM) method.

\section{RT-PCR detection of mRNA expression levels}

IPEC-J2 cells treated with MMSC or untreated cells in 6well plates were cultured for $24 \mathrm{~h}$. Total RNA of the IPEC-J2 cells was extracted from the cells using Trizol ${ }^{\circledR}$ reagent (Invitrogen). RNA was reversely transcribed into cDNA using the TaKaRa reverse transcription kit (TaKaRa). Real-time polymerase chain reaction (RT-PCR) was performed by using FastStart Universal SYBR $\AA$ Green Master (Rox) (Roche). RT-PCR amplification procedure was as follows: initial denaturation for 10 minutes at $95^{\circ} \mathrm{C}$ followed by 35 cycles, with each cycle consisting of 30 seconds at $95^{\circ} \mathrm{C}$, 30 seconds at $60^{\circ} \mathrm{C}$, in addition to a final extension for 5 minutes at $72^{\circ} \mathrm{C}$. The amplification reactions with $\beta$-actin as internal reference and three pairs of target primers were listed in Table 1. The relative expression amount was calculated by using equation $2^{-\triangle \Delta C T}$ (Livak and Schmittgen 2001). Each sample and negative controls (no template) were run in triplicate.

\section{Western blotting detection of protein expression}

Western blotting (WB) analysis was performed to evaluate the protein expression. Briefly, IPEC-J2 cells in 6-well plates treated with MMSC in 6-well plates were washed one time with PBS and then total proteins of IPEC-J2 cells were extracted by using the radioimmunoprecipitation (RIPA) lysis buffer (NCM Biotech) supplement with 1\% protease inhibitor (Solarbio). $1 \mathrm{ml}$ lysis buffer was added to the well, lysed for 30 minutes on ice, then collected and centrifuged at 14,000 $\times \mathrm{g}$ for 20 minutes. Supernatant fractions contained total proteins. The protein concentration of supernatant fractions was quantified by a standard bicinchoninic acid (BCA) protein assay (Pierce). An equal amount of protein was separated on SDS-PAGE gels and then transferred onto PVDF membranes. PVDF membranes were blocked with

Table 1: Sequence of primers used for detection of genus Brucella.

\begin{tabular}{lllr}
\hline Gene name & Primer & Sequence $\left(5^{\prime}-3^{\prime}\right)$ & PCR products \\
\hline$\beta$-actin & Forward & CTCCATCATGAAGTGCGACG & $242 \mathrm{bp}$ \\
& Reverse & CCTGCTTGCTGATCCACATC & $176 \mathrm{bp}$ \\
Zo-1 & Forward & ATCTCGGAAAATGCCAGGA & $180 \mathrm{bp}$ \\
& Reverse & CCTTCCCCTCAGAAACCCAT & $193 \mathrm{bp}$ \\
& Forward & TGGAAGATGATGAGGTGCAGA & 10 \\
& Reverse & CAGTGAAGAGAGCCTGACCA & \\
\hline
\end{tabular}


$5 \%$ non-fat dry milk in PBST at $37^{\circ} \mathrm{C}$ for 2 hours. After six times washing with PBST, membranes were incubated with primary antibody at $4^{\circ} \mathrm{C}$ overnight. The antibodies used in our study including rabbit polyclonal anti-Occludin $(1: 1000$; Proteintech), rabbit polyclonal anti-Claudin-1 (1:1000; Proteintech), rabbit polyclonal anti-Zo-1 (1:1000; Proteintech), mouse anti- $\beta$ actin (1:500; Boster). After six times washing with PBST, secondary antibody, such as antirabbit IgG, anti-mouse IgG (1:5000, Jackson ImmunoResearch), was added and incubated at $37^{\circ} \mathrm{C}$ for 30 minutes. Finally, membranes were examined with ECL reagent (NCM Biotech) followed by analysis using Gel Doc TM XR (Bio-rad). The intensity of the blotting was quantified using qualityone software.

\section{Statistical analysis}

Statistical analysis was performed using GraphPad Prism version 8.00 (GraphPad Software). The comparison among groups was analyzed by one-way ANOVA. Data were shown as the mean \pm SEM. Statistical significance was determined at $\left.\left.p<0.05^{(*)}, p<0.01\left(^{* *}\right), p<0.001^{* * *}\right), p<0.0001^{(* * *}\right)$.

\section{RESULTS AND DISCUSSION \\ Cytotoxicity of MMCS on IPEC-J2 cells}

To estimate cytotoxic effect of MMCS, CCK-8 assay was used to evaluate the IPEC-J2 cells proliferation. As shown in Table 2, compared with control group, the highest level of IPEC-J2 cells proliferation was detected in group $2(0.5 \mathrm{mM})$, whereas no significant difference was observed between group $2(0.5 \mathrm{mM})$ and group $3(1 \mathrm{mM})$. Cells treated with three different concentrations of MMSC showed high cell viability, indicating that MMCS is nontoxic to IPEC-J2 cells.

\section{Effects of MMSC on IPEC-J2 cells apoptosis}

FCM method was performed to detect IPEC-J2 cells apoptosis. As shown in Fig 1, cell survival was highest in group $2(0.5 \mathrm{mM})$, reaching to $97.02 \%$, followed by $95.74 \%$ in group $3(1 \mathrm{mM})$ and $94.75 \%$ in group $1(0.1 \mathrm{mM})$, while $93.42 \%$ in control group. As shown in Table 3, MMCS would significantly reduce early and late cell apoptosis of IPEC-J2 cells $(p<0.05)$, comparing with untreated cells. There was no dose-dependent of MMCS on inhibition of IPEC-J2 cells apoptosis, but $0.5 \mathrm{mM}$ MMCS showed best effect on inhibition of cells apoptosis. Detailed information was summarized in Table 3.

\section{Analysis of mRNA expression levels of tight junction genes in IPEC-J2 cells}

RT-PCR was conducted to determine the mRNA expression levels of tight junction genes, including occludin, claudin-1 and Zo-1. As shown in Fig 2a, the addition of 0.5 mM MMCS and $1 \mathrm{mM}$ MMCS to the culture medium both significantly increased the mRNA expression of occludin in comparison with the mRNA expression in the control group. Interestingly, when IPEC-J2 cells were treated with $0.5 \mathrm{mM} \mathrm{MMCS}$, the mRNA expression level of claudin-1 and Zo-1 would significantly improve $(p<0.01)$, but not observed in the group 3 (1 mM MMCS) (Fig 2b and Fig 2c). Moreover, when IPEC$\mathrm{J} 2$ cells treated with $0.1 \mathrm{mM}$ MMCS, the mRNA expression of these three tight junction genes showed no difference with those in control group (Fig 2). These results indicated that there was a dose-dependent effect of MMSC in stimulating mRNA expression and the $0.5 \mathrm{mM}$ MMCS showed best effect on increasing the mRNA expression of tight junction genes.

\section{Analysis of protein expression levels of tight junction genes in IPEC-J2 cells}

For estimating effect of MMCS on protein expression levels, WB analysis was further used to evaluate tight junction proteins expression. As shown in Fig 3, in comparison with the control group, three different concentrations $(0.1 \mathrm{mM}$, $0.5 \mathrm{mM}$ and $1 \mathrm{mM}$ ) of MMSC significantly increased the protein expression of occludin $(p<0.01, p<0.0001, p<0.001)$. The protein expression of claudin-1 significantly improved in $0.5 \mathrm{mM} \mathrm{MMSC}(p<0.0001)$ and $1 \mathrm{mM} \mathrm{MMSC}(p<0.001)$. However, only $0.5 \mathrm{mM}$ MMSC was added into the medium, the relative protein abundance of Zo-1 significantly improved $(p<0.001)$, but not observed in the $0.1 \mathrm{mM}$ MMSC and 1 mM MMSC group. These results implied that MMCS supplemented may stimulate tight junction proteins expression and the $0.5 \mathrm{mM}$ MMCS exhibited a higher level of protein expression.

Table 2: Effects of different concentrations of MMCS on IPEC-J2 cells proliferation.

\begin{tabular}{lcccc}
\hline & Control & $0.1 \mathrm{mM}$ & $0.5 \mathrm{mM}$ & $1 \mathrm{mM}$ \\
\cline { 2 - 5 } Cell proliferation activity (\%) & $91.91 \pm 6.25^{\mathrm{c}}$ & $107.30 \pm 7.29^{\mathrm{b}}$ & $118.7 \pm 1.87^{\mathrm{a}}$ & $112.77 \pm 13.12^{\mathrm{ab}}$ \\
\hline
\end{tabular}

Note: Results were presented as means \pm SEM of triplicate observations. Different lowercase letters in the same column denoted statistically significant difference $(p<0.05)$ and the same letters indicated no significant difference between the two groups $(p>0.05)$.

Table 3: Results of MMCS on cell cycle of IPEC-J2 cells.

\begin{tabular}{lcccc}
\hline & Control & $0.1 \mathrm{mM}$ & $0.5 \mathrm{mM}$ & $1 \mathrm{mM}$ \\
\hline Late cell apoptosis (\%) & $2.28 \pm 0.13^{\mathrm{a}}$ & $1.67 \pm 0.08^{\mathrm{b}}$ & $0.69 \pm 0.05^{\mathrm{c}}$ & $1.13 \pm 0.11^{\mathrm{d}}$ \\
Early cell apoptosis (\%) & $4.09 \pm 0.19^{\mathrm{a}}$ & $3.20 \pm 0.08^{\mathrm{b}}$ & $2.13 \pm 0.11^{\mathrm{c}}$ & $2.62 \pm 0.15^{\mathrm{d}}$ \\
\hline
\end{tabular}

Note: Results were presented as means \pm SEM of triplicate observations. Different lowercase letters in the same column denoted statistically significant difference $(p<0.05)$ and the same letters indicated no significant difference between the two groups $(p>0.05)$. 

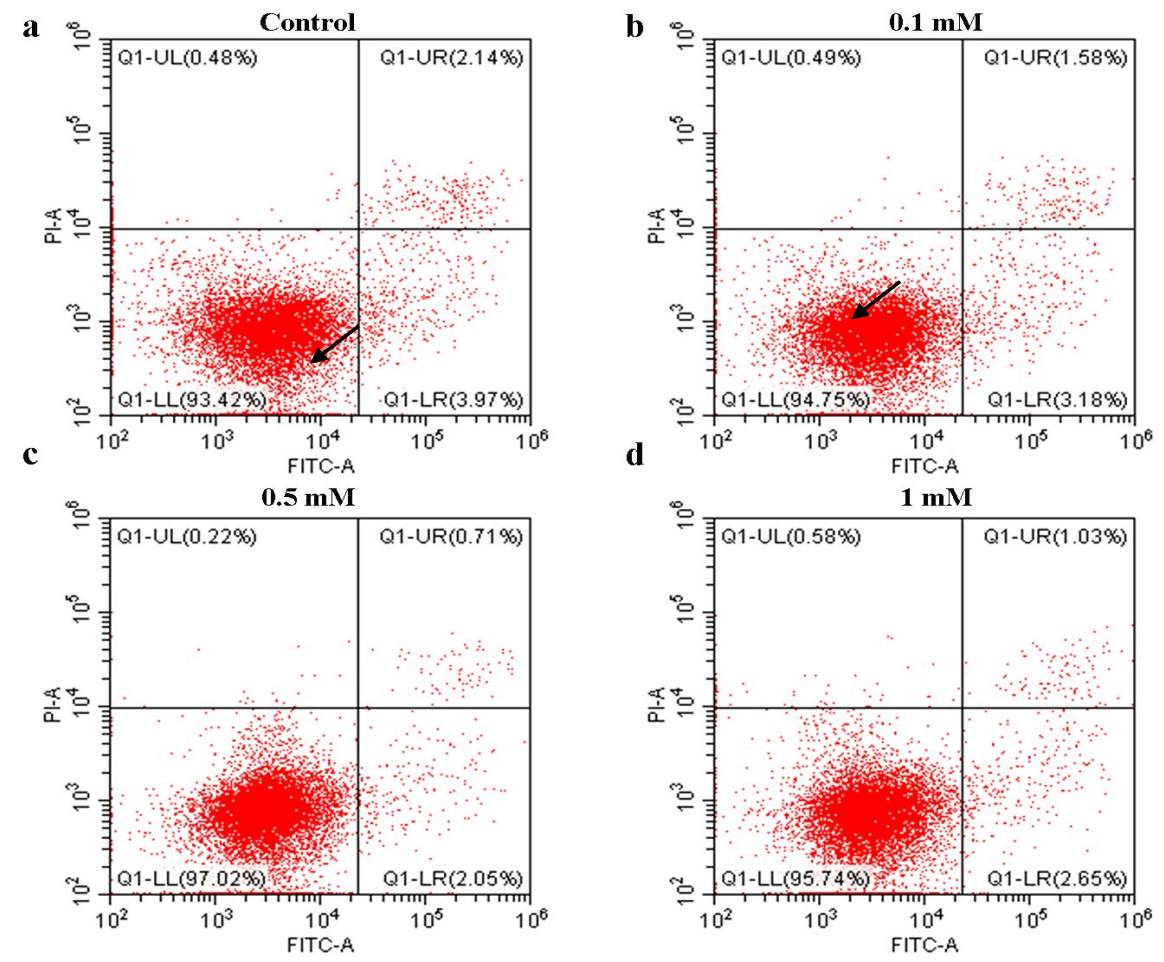

Fig 1: Effects of different concentrations of MMCS on IPEC-J2 cells apoptosis.

IPEC-J2 cells treated with MMCS in 6-well plates were collected, stained with the Annexin V/PI Apoptosis Detection kit and analyzed by FCM. Untreated IPEC-J2 cells were used as the control. a untreated IPEC-J2 cells; b IPEC-J2 cells treated with 0.1 mM MMCS; c IPEC-J2 cells treated with $0.5 \mathrm{mM}$ MMCS; d IPEC-J2 cells treated with $1 \mathrm{mM}$ MMCS. UL region was merornecrosis (upper left), LL region was cell survival (lower left), UR region was late cell apoptosis (upper right) and LR region was early cell apoptosis (lower right).

Intestinal microbiology and health of the mammals play an important role in the digestion and absorption of nutrients, growth of animals, improve immunity and disease resistance and so on (Li et al. 2019, Yatsunenko et al. 2012). In recent years, along with the application of antibiotics abuse, intestinal health problems in livestock and poultry are becoming increasingly serious, impacting the production performance and economic benefits of animal husbandry industry. Intestinal permeability was closely to prevent harmful substances from invading and important in animal subjects with diarrheal disease (Boudry et al. 2004). Therefore, there has been an increasing scientific interest on the use of nutritional manipulations or other mechanisms to improve the intestinal barrier function of young animals.

Amino acids or trace elements are indispensable for intestinal health and biological functions, which affected the intestinal mucosal barrier by regulating the expression of intestinal cytokines and tight junction proteins (Tretola et al. 2020, Wu 2009, Xia et al. 2016). Occludin, claudin-1 and Zo-1 were considered to be the key proteins of tight junction integrity and controlled the function and permeability of the intestinal (Brun et al. 2007). It is reported that $\mathrm{Zn}$ supplementation in dietary treatments would reduce intestinal permeability by enhancing occludin and Zo-1 expression (Sturniolo et al. 2002, Zhang and Guo 2009). Reducing arginine concentration was feasible in IPEC-J2 cells, which guaranteed nutrient uptake and cell barrier function by improving the occludin and claudin-1 expression (Xia et al. 2016). Moreover, studies showed that added dietary threonine would benefit to growth performance, health, immunity and gastrointestinal function of weaning pigs (Trevisi et al. 2015).

In the present study, different concentrations of MMSC were chosen to evaluate its effect on activity and tight junction protein expression of IPEC-2 cells. As shown in Table 2 and Table 3, MMCS is nontoxic to IPEC-J2 cells and cells treated with three different concentrations of MMSC showed high cell viability and low cells apoptosis (Fig.1). As tight junction protein and adherent junction are important in the maintenance of epithelial integrity, we further analyzed mRNA expression and protein expression of tight junction protein in IPEC-J2 cells. Results from RT-PCR showed that when IPEC-2 cells treated with 0.5 mM MMSC, mRNA levels of tight junction genes, including occludin, claudin-1 and Zo-1 were significantly increased $(P<0.001, P<0.01$ and $\mathrm{P}<0.01$ ) (Fig 2). Whereas higher MMSC supplemented (1 $\mathrm{mM})$, there were no differences compared to the control group. Similarly, this phenomenon existed in protein expression levels of tight junction proteins. As shown in Fig. 3, $0.5 \mathrm{mM}$ MMSC significantly enhanced protein expression of occludin, claudin-1 and Zo-1. Excepting Zo-1, $1 \mathrm{mM}$ MMSC treated with IPEC-J2 cells, would stimulate occludin, 
a

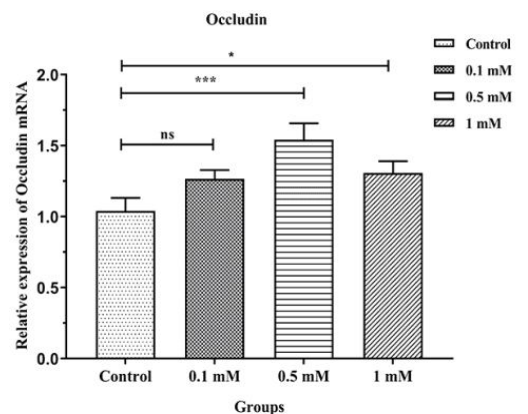

b

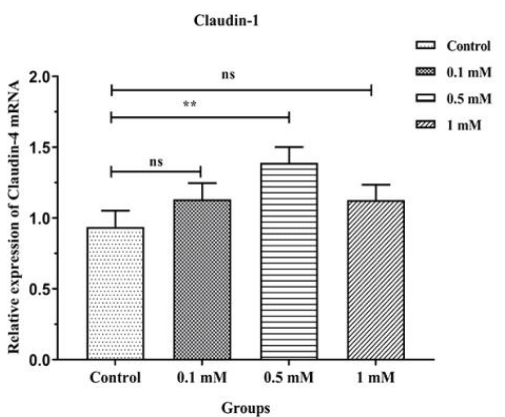

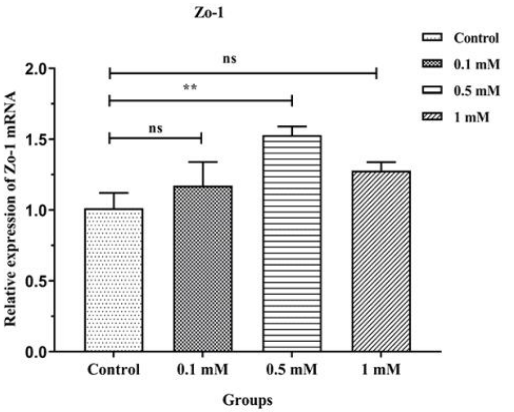

Fig 2: RT-PCR results of mRNA expression of tight junction genes.

IPEC-J2 cells in 6-well plates were extracted using Trizol ${ }^{\circledR}$ reagent and RT-PCR was used to analyze the mRNA expression levels of tight junction genes. a Occludin; b Claudin-1; c Zo-1. Relative gene expression was evaluated using the $2^{-\Delta \triangle C T}$ method and $\beta$-actin was used as internal reference. The data above were representative of three independent experiments and error bars indicated the $\operatorname{SEM}(n=3)$. Statistical significance was determined at $p<0.05\left(^{*}\right), p<0.01\left(^{* *}\right), p<0.001\left(^{* * *}\right), p<0.0001\left(^{* * * *}\right)$.

a

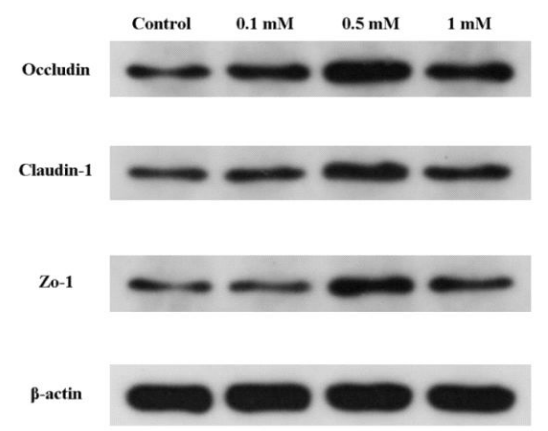

b

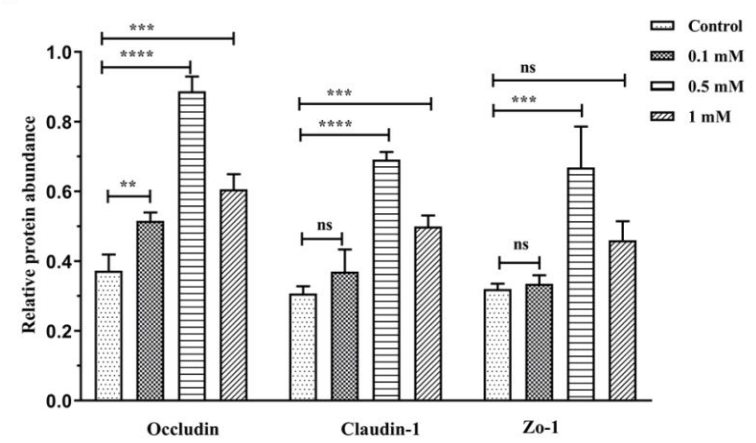

Fig 3: Effects of different MMCS concentrations on tight junction proteins expression.

a Results from WB anaysis; b Relative protein expression levels of tight junction proteins. The value of protein expression=densitometry units of target protein/densitometry units of $\beta$-actin protein detected by WB. Error bars indicated the SEM $(n=3)$ and statistical significance was determined at $p<0.05\left({ }^{*}\right), p<0.01\left(^{* *}\right), p<0.001\left(^{* * *}\right), p<0.0001\left(^{* * * *}\right)$.

claudin-1 protein expression. These data above demonstrated that MMCS, within the suitable concentration, could enhance occludin, claudin-1 and Zo-1 expression in IPEC-2 cells. It indicated that MMCS may play a role in regulating intestinal permeability and barrier function of intestinal mucosal epithelial cells.

In summary, MMCS is nontoxic to IPEC-J2 cells and has a function in promoting the mRNA and proteins expressions of the key tight junction proteins (occludin, claudin-1 and Zo-1). We found that 0.5 mM MMCS exhibited a higher level of mRNA and protein expression in IPEC-J2 cells.

\section{CONCLUSION}

The present study investigated the effects of extracellular MMSC on intestinal epithelial cell line (IPEC-J2). The results showed that MMSC could stimulate IPEC-J2 cells proliferation and inhibit cell apoptosis. In addition, the realtime quantitative polymerase chain reaction (RT-PCR) and western blotting $(\mathrm{WB})$ results indicated that $0.5 \mathrm{mM}$ MMSC significantly increased the mRNA and protein expression of tight junction genes, including occludin, claudin-1 and zonula occludin-1 (Zo-1). These findings may provide valuable information to further investigate the possible mechanism and function of MMCS on the intestinal barrier function.

\section{ACKNOWLEDGEMENT}

This work was supported by Key Projects of Science and Technology Department of Henan Province (202102110086 and 182102410030) and Innovation Team Project of Henan University of Animal Husbandry and Economy (24030015) and Key Scientific Research Project of Colleges and Universities in Henan Province (project number: 18A230006) and Fund Support for Doctoral Research of Henan University of Animal Husbandry and Economy (2018HNUAHEDF026).

\section{Conflicts of interest}

The authors declare that they have no conflicts of interest. 


\section{REFERENCES}

Barrett, K.E. (2008). New ways of thinking about (and teaching about) intestinal epithelial function. Adv. Physiol. Educ. 32(1): 25-34.

Boudry, G., Peron, V., Le Huerou-Luron, I., Lalles, J. P. and Seve, B. (2004). Weaning induces both transient and longlasting modifications of absorptive, secretory and barrier properties of piglet intestine. J. Nutr. 134(9): 2256-62.

Brun, P., Castagliuolo, I., Di Leo, V., Buda, A., Pinzani, M., Palu, G. and Martines, D. (2007). Increased intestinal permeability in obese mice: New evidence in the pathogenesis of nonalcoholic steatohepatitis. Am. J. Physiol Gastrointest Liver Physiol. 292(2): G518-25.

Farkas, A.E., Capaldo, C.T. and Nusrat, A. (2012). Regulation of epithelial proliferation by tight junction proteins. Ann. N. Y. Acad. Sci. 1258: 115-24.

Gezginci-Oktayoglu, S., Turkyilmaz, I. B., Ercin, M., Yanardag, R. and Bolkent, S. (2016). Vitamin $U$ has a protective effect on valproic acid-induced renal damage due to its antioxidant, anti-inflammatory and anti-fibrotic properties. Protoplasma. 253(1): 127-35.

Guevarra, R.B., Hong, S.H., Cho, J.H., Kim, B.R., Shin, J., Lee, J.H., Kang, B.N., Kim, Y.H., Wattanaphansak, S., Isaacson, R.E., Song, M. and Kim, H.B. (2018). The dynamics of the piglet gut microbiome during the weaning transition in association with health and nutrition. J. Anim. Sci. Biotechnol. 9: 54.

Ichikawa, T., Ito, Y., Saegusa, Y., Iwai, T., Goso, Y., Ikezawa, T. and Ishihara, K. (2009). Effects of combination treatment with famotidine and methylmethionine sulfonium chloride on the mucus barrier of rat gastric mucosa. J Gastroenterol Hepatol. 24(3): 488-92.

Kim, W.S., Seo, H.M., Kim, W.K., Choi, J.S., Kim, I. and Sung, J.H. (2015). Thephotoprotective effect of S-Methylmethionine sulfonium in skin. Int. J. Mol. Sci. 16(8): 17088-100.

Kojima, T., Yamamoto, T., Murata, M., Chiba, H., Kokai, Y. and Sawada, N. (2003). Regulation of the blood-biliary barrier: interaction between gap and tight junctions in hepatocytes. Med. Electron. Microsc. 36(3): 157-64.

Li, W., Deng, Y., Chu, Q. and Zhang, P. (2019). Gut microbiome and cancer immunotherapy. Cancer Lett, 447: 41-47.

Liu, F., Li, G., Wen, K., Bui, T., Cao, D., Zhang, Y. and Yuan, L. (2010). Porcine small intestinal epithelial cell line (IPECJ2) of rotavirus infection as a new model for the study of innate immune responses to rotaviruses and probiotics. Viral Immunol. 23(2): 135-49.

Livak, K.J. and Schmittgen, T.D. (2001). Analysis of relative gene expression data using real-time quantitative PCR and the 2(-Delta Delta $\mathrm{C}(\mathrm{T})$ ) method. Methods. 25(4): 402-8.

Oztay, F., Tunali, S., Kayalar, O. and Yanardag, R. (2020). The protective effect of vitamin $U$ on valproic acid-induced lung toxicity in rats via amelioration of oxidative stress. J. Biochem. Mol. Toxicol. 34(12): e22602.

Roehlen, N., Roca Suarez, A.A., El Saghire, H., Saviano, A., Schuster, C., Lupberger, J. and Baumert, T. F. (2020). Tight Junction Proteins and the Biology of Hepatobiliary Disease. Int. J. Mol. Sci. 21(3).
Salim, A.S. (1992). Role of sulfhydryl-containing agents in the healing of erosive gastritis and chronic gastric ulceration in the rat. J. Pharm. Sci. 81(1): 70-3.

Sturniolo, G.C., Fries, W., Mazzon, E., Di Leo, V., Barollo, M. and D'Inca, R. (2002). Effect of zinc supplementation on intestinal permeability in experimental colitis. J. Lab. Clin. Med. 139 (5): 311-5.

Suzuki, T. and Hara, H. (2009). Quercetin enhances intestinal barrier function through the assembly of zonula [corrected] occludens-2, occludin and claudin-1 and the expression of claudin-4 in Caco-2 cells. J. Nutr. 139(5): 965-74.

Tretola, M., Bee, G. and Silacci, P. (2020). Gallic acid affects intestinal-epithelial-cell integrity and selected amino-acid uptake in porcine in vitro and ex vivo permeability models. Br. J. Nutr: 1-9.

Trevisi, P., Corrent, E., Mazzoni, M., Messori, S., Priori, D., Gherpelli, Y., Simongiovanni, A. and Bosi, P. (2015). Effect of added dietary threonine on growth performance, health, immunity and gastrointestinal function of weaning pigs with differing genetic susceptibility to Escherichia coli infection and challenged with E. coli K88ac. J. Anim. Physiol. Anim. Nutr. (Berl). 99(3): 511-20.

Wu, G. (2009). Amino acids: Metabolism, functions and nutrition. Amino Acids. 37(1): 1-17.

Xia, M., Ye, L., Hou, Q. and Yu, Q. (2016). Effects of arginine on intestinal epithelial cell integrity and nutrient uptake. $\mathrm{Br}$. J. Nutr. 1-7.

Yang, Q., Huang, X., Wang, P., Yan, Z., Sun, W., Zhao, S. and Gun, S. (2019). Longitudinal development of the gut microbiota in healthy and diarrheic piglets induced by age-related dietary changes. Microbiologyopen. 8(12): e923.

Yatsunenko, T., Rey, F.E., Manary, M.J., Trehan, I., DominguezBello, M.G., Contreras, M., Magris, M., et al. (2012). Human gut microbiome viewed across age and geography. Nature. 486(7402): 222-7.

Yu, Y.B. and Li, Y.Q. (2014). Enteric glial cells and their role in the intestinal epithelial barrier. World J. Gastroenterol. 20(32): 11273-80.

Zakrzewski, S.S., Richter, J.F., Krug, S. M., Jebautzke, B., Lee, I.F., Rieger, J., Sachtleben, M., Bondzio, A., Schulzke, J.D., Fromm, M. and Gunzel, D. (2013). Improved cell line IPEC-J2, characterized as a model for porcine jejunal epithelium. PLoS One. 8(11): e79643.

Zhang, B. and Guo, Y. (2009). Supplemental zinc reduced intestinal permeability by enhancing occludin and zonula occludens protein-1 (ZO-1) expression in weaning piglets. Br. J. Nutr. 102(5): 687-93.

Zong, Q.F., Huang, Y.J., Wu, L.S., Wu, Z.C., Wu, S.L. and Bao, W.B. (2019). Effects of porcine epidemic diarrhea virus infection on tight junction protein gene expression and morphology of the intestinal mucosa in pigs. Pol. J. Vet. Sci. 22(2): 345-353. 